\title{
Análisis morfométrico de semilla y desarrollo de plántulas de maguey sacatoro (Agave angustifolia Haw.)
}

\section{Morphometric analysis of seed and seedling development of maguey sacatoro (Agave angustifolia Haw.)}

\begin{abstract}
Elías Hernández-Castro 1 (D)
Yoccira Yanet López-Sandoval 1 (D) José Luis Escobar-Álvarez ${ }^{3}$ [D,

Omar Ramírez-Reynoso ${ }^{2}$ (D)

María de los Ángeles Maldonado-

Peralta 4 (D)

José Luis Valenzuela-Lagarda $2 *$ (D)

${ }^{1}$ Facultad de Ciencias Agropecuarias

y Ambientales, Unidad Tuxpan.

Carretera Iguala-Tuxpan, km 2.5 .

Iguala de la Independencia, Guerrero,

México.

${ }^{2}$ Centro Regional de Educación

Superior de la Costa Chica, UA-

Gro. Florencio Villarreal, CP. 41800.

Guerrero, México.

${ }^{3}$ Postgrado en Recursos Genéti-

cos y Productividad, Colegio de

Postgraduados, Carretera México-

Texcoco km. 36.5, CP. 56230. Mon-

tecillo, Texcoco, Estado de México,

México.

${ }^{4}$ Facultad de Medicina Veterinaria

y Zootecnia No. 2, Universidad

Autónoma de Guerrero, Av. Carretera

Acapulco-Pinotepa Nacional, Km 197,

CP. 41940. Cuajinicuilapa, Guerrrero,

México.
\end{abstract}

*Autor de correspondencia:

joseluislagarda@uagro.mx

Artículo científico

Recibido: 04 de marzo 2021

Aceptado: 16 de diciembre 2021

Como citar: Hernández-Castro E, López-Sandoval YY, Escobar-Álvarez JL, Ramírez-Reynoso O, MaldonadoPeralta MA, Valenzuela-Lagarda JL (2021) Análisis morfométrico de semilla y desarrollo de plántulas de maguey sacatoro (Agave angustifolia Haw.). Ecosistemas y Recursos Agropecuarios 8(3): e2964. DOI: 10.19136/era.a8n3.2964
RESUMEN. Los Agaves son nativos de América y tienen diferentes usos, entre los que destacan la obtención de fibras y elaboración de bebidas alcohólicas; esto ha ocasionado una considerable disminución en las poblaciones silvestres; por lo que, el objetivo fue caracterizar morfológicamente cápsulas y semillas, y evaluar el desarrollo de la plántula de maguey sacatoro (Agave angustifolia Haw.) colectadas en diferentes sitios del centro y norte del estado de Guerrero, México, para su propagación por semilla. Para el análisis morfométrico se empleó un diseño de un solo factor (Localidad) y para las variables de calidad de semilla, un análisis bifactorial (localidad y tiempo de inmersión). Los resultados obtenidos se sometieron a análisis de varianza y pruebas de medias (Tukey, $p \leq 0.05$ ). Se tomaron peso, altura y ancho de las cápsulas y semillas. Se realizó la prueba de germinación estándar y la viabilidad con la prueba del tetrazolio. Posteriormente, se evaluó el desarrollo de plántula; en tres tiempos de inmersión en agua (0,24 y 48 h) y se realizaron mediciones en raíz, hoja y tallo. Motuapa presentó cápsulas con mayor número de semillas y semillas normales (241 y 140, respectivamente). Las cápsulas de mayor longitud la presentaron Atetetla, Motuapa y los Amates (5.37 \pm $0.50,5.10 \pm 0.40$ y $4.97 \pm 0.36 \mathrm{~cm}$, respectivamente), Ateteta tuvo mayor ancho de cápsula $(2.63 \pm 0.05 \mathrm{~cm})$, germinación $(95 \%)$ y viabilidad $(89.4 \%)$. Por lo que, La producción de plántula de $A$. sacatoro, mediante el uso de semillas puede ser una alternativa viable.

Palabras clave: Agave, cápsulas, reproducción sexual, semillas, tetrazolio.

ABSTRACT. Agaves are native to America and have different uses, among which the obtaining of fibers and the elaboration of alcoholic beverages stand out; This has caused a considerable decline in wild populations; Therefore, the objective was to morphologically characterize capsules and seeds, and evaluate the development of the maguey sacatoro (Agave angustifolia Haw.) seedling collected in different sites in the center and north of the state of Guerrero, Mexico, for their propagation by seed. For the morphometric analysis a single factor design (Locality) was used and for the seed quality variables, a bifactorial analysis (locality and immersion time). The results obtained were subjected to analysis of variance and tests of means (Tukey, $p \leq 0.05$ ). Weight, height and width of the capsules and seeds were taken. The standard germination test and viability were performed with the tetrazolium test. Subsequently, the seedling development was evaluated; in three times of immersion in water $(0,24$ and $48 \mathrm{~h})$ and measurements were made in root, leaf and stem. Motuapa presented capsules with a higher number of seeds and normal seeds (241 and 140, respectively). The longest capsules were presented by Atetetla, Motuapa and Los Amates $(5.37 \pm 0.50,5.10 \pm 0.40$ and $4.97 \pm 0.36 \mathrm{~cm}$, respectively), Ateteta had greater capsule width $(2.63 \pm 0.05 \mathrm{~cm})$, germination (95\%) and viability (89.4\%). Therefore, the production of $A$. sacatoro seedling, through the use of seeds, can be a viable alternative.

Key words: Agave, capsules, seeds, sexual reproduction, tetrazolium. 


\section{INTRODUCCIÓN}

Las plantas del género Agave son originarias de América y se tienen descritas 273 especies, desde el Norte de EE.UU. hasta Bolivia y Paraguay (ZúñigaEstrada 2013). México, es el país con mayor riqueza y diversidad de agaves, donde se tienen reportadas 205, de las cuales 151 son especies endémicas (Esparza-lbarra et al. 2015). Al respecto, FloresMaya et al. (2015) señalan que la mayor diversidad se encuentra en la parte central de México, Valle de Tehuacán-Cuicatlán, cañón barranca de Meztitlán, Sierra Madre Occidental y en regiones del desierto de Chihuahua, el cual podría ser el centro de origen del género. Este grupo de plantas es valorado por su importancia social, cultural, ecológica y económica (Barrientos-Rivera et al. 2019). Comúnmente se le conoce como maguey o mezcal y se aprovecha como fuente de productos alimenticios, ornamental, medicinal, material para construcción y bebidas destiladas como el tequila, sotol, pulque y mezcal, entre otros (Esparza-lbarra et al. 2015, Barrientos-Rivera et al. 2019).

Este género de plantas, se encuentra en ambientes diversos que incluyen bosques de coníferas en sistemas montañosos y matorrales desérticos costeros característicos de las zonas áridas, en altitudes que van desde los 0 hasta los 3400 msnm (RamírezTobías et al. 2014, Vázquez-Acosta et al. 2020), crecen en sitios con limitaciones de humedad, altas temperaturas (Candia-Acosta et al. 2019) y presentan alta tolerancia ecológica; esto se debe, a que han adoptado mecanismos fisiológicos que les ha permitido una adaptación local en hábitats heterogéneos (Barrientos-Rivera et al. 2019).

La propagación mediante hijuelos es la forma más común de reproducción en Agaves (VázquezDíaz et al. 2011), los hijuelos se mantienen uniformes genéticamente y el porcentaje en el trasplante es mayor en relación con la propagación por semilla (Arzate-Fernández et al. 2016). Mientras que, los individuos originados por semillas mantienen la estructura y dinámica de las poblaciones de maguey (Ramírez-Tobías et al. 2012) y presentan incremento en la variabilidad genética (Ortiz-Hernández et al.
2018). La forma principal de reproducción de este género es la asexual, al producir clones en distintas partes de la roseta o inflorescencia; mientras que, la sexual se logra mediante la polinización realizada por murciélagos e insectos (García-Mendoza 2007).

En el estado de Guerrero, Villaseñor (2016) reportó que se tienen registradas 16 especies del género Agave y de las que, $A$. cupreata Trel. et Bergel (maguey papalote o ancho) y $A$. angustifolia Haw. (maguey mezcalero o espadín) se utilizan principalmente para la extracción de materia prima para la elaboración de mezcal. Esto ha provocado presión sobre las poblaciones silvestres; por lo que, los productores han buscado alternativas para la reproducción de esta especie (Huerta-Zavala et al. 2019). Por ello, el objetivo del presente trabajo fue caracterizar morfológicamente cápsula y semillas, y evaluar el desarrollo de la plántula de maguey sacatoro colectada en diferentes sitios del norte del estado de Guerrero.

\section{MATERIALES Y MÉTODOS}

\section{Área de estudio}

Los sitios de colecta comprendieron cuatro municipios del estado de Guerrero (Tabla 1). Se seleccionaron dos plantas por localidad, los criterios de selección fueron: plantas sanas libres de plagas o enfermedades, vigorosas (color verde fuerte), de buen porte (altura y área foliar de planta) superior a la media de la población. Se cortó el quiote a un metro de altura a partir del centro del tallo, de las panículas, se seleccionaron al azar, cinco cápsulas cerradas por planta, se depositaron en bolsas de papel y se trasladaron al Laboratorio de Nutrición y Fisiología Vegetal, ubicado en el Campus Tuxpan de la Facultad de Ciencias Agropecuarias y Ambientales de la Universidad Autónoma de Guerrero, México. Las cápsulas se secaron a temperatura ambiente promedio de $33{ }^{\circ} \mathrm{C}$, durante 20 días y; posteriormente, se realizó una depuración, en forma visual, de las semillas que no presentaron endospermo ni embrión (semillas estériles). 
Tabla 1. Ubicación geográfica de las localidades donde se realizaron las colectas de semilla de $A$. aungustifolia Haw. del estado de Guerrero.

\begin{tabular}{|c|c|c|c|c|c|}
\hline Municipio & Localidad & $\begin{array}{l}\text { Fecha de } \\
\text { colecta }\end{array}$ & $\begin{array}{l}\text { Coordenadas } \\
\text { geográficas }\end{array}$ & $\begin{array}{r}\text { Altitud } \\
(\mathrm{msnm})\end{array}$ & $\begin{array}{r}\text { Temperatura } \\
\text { promedio }\left({ }^{\circ} \mathrm{C}\right)\end{array}$ \\
\hline Ahuacoutzingo & Motuapa & $27 /$ sep/16 & $\begin{array}{l}17^{\circ} 44^{\prime} 57.3^{\prime \prime} \text { LN } \\
99^{\circ} 02^{\prime} 16.2^{\prime \prime} \text { LO }\end{array}$ & $\begin{array}{l}1 \\
312\end{array}$ & 24 \\
\hline Huitzuco & Atetetla & $01 /$ oct/16 & $\begin{array}{l}18^{\circ} 19^{\prime} 74.4^{\prime \prime} \text { LN } \\
99^{\circ} 23^{\prime} 85.6^{\prime \prime} \text { LO }\end{array}$ & 876 & 26 \\
\hline Chilapa & Los Amates & $21 /$ oct $/ 16$ & $\begin{array}{l}17^{\circ} 32^{\prime} 0.19^{\prime \prime} \text { LN } \\
99^{\circ} 11^{\prime} 79.9^{\prime \prime} \text { LO }\end{array}$ & $\begin{array}{l}1 \\
625\end{array}$ & 24 \\
\hline Atenango del Río & Coacán & 05/nov/16 & $\begin{array}{l}18^{\circ} 10^{\prime} 40.3^{\prime \prime} \text { LN } \\
99^{\circ} 12^{\prime} 19.7^{\prime \prime} \text { LO }\end{array}$ & 960 & 26 \\
\hline
\end{tabular}

\section{Variables morfométricas}

Se cuantificó el número total de semillas por cápsula, el largo (LC) y ancho de cápsula (AC), largo (LS) y ancho de semilla (AS) con un vernier digital (HER-411, Steren ${ }^{\circledR}$ ) y se expresó en $\mathrm{cm}$. El peso de cada cápsula y peso de 1000 semillas se determinó con una balanza analítica Santorius entris $\left.{ }^{\circledR}\right)(2241$ 1S) y se reportó en gramos, la estimación se realizó de acuerdo a lo establecido por ISTA (1996).

\section{Germinación y viabilidad de semillas}

Se realizó una prueba de germinación estándar, mediante la metodología propuesta por el ISTA (2014) y se determinaron los porcentajes de semillas germinadas y la viabilidad con prueba del tetrazolio al 1\% (Maldonado-Peralta et al. 2016).

\section{Desarrollo de plántula}

Para evaluar el desarrollo de plántula, las semillas se sometieron a tres tratamientos 1) siembra directa, 2) inmersión durante 24 h y 3) inmersión durante $48 \mathrm{~h}$ en agua destilada. La siembra se realizó en vasos de plástico de poliestireno \# 10 y el sustrato utilizado fue peat moss $\left(B M 2{ }^{\circledR}\right)$ y agrolita $(8: 2$ $\mathrm{v} / \mathrm{v}$ ) con 80 unidades experimentales por tratamiento. Los datos se tomaron cada dos meses después de la siembra por un periodo de seis meses y se evaluaron en cm, altura de planta (AP), y largo (LH) y ancho de hoja $(\mathrm{AH})$.

\section{Análisis de datos}

Los datos obtenidos de las variables morfométricas, se analizaron bajo un diseño de un factor (localidad) con cuatro niveles (Atetetla, Coacán, Los
Amates y Motuapa), mientras que, para las variables del desarrollo de plántula, se estableció un diseño completamente al azar con arreglo bifactorial, donde un factor fue localidades con cuatro niveles (Atetetla, Coacán, Los Amates y Motuapa) y el otro factor fue tiempo de remojo de las semillas con tres niveles ( 0 , 24 y 48 h). Los porcentajes de germinación y viabilidad se transformaron obteniendo el arcoseno de su raíz cuadrada y dividiéndolos entre 100 a fin de homogenizar la varianza. Posteriormente, los datos de variables morfométricas, germinación y viabilidad, y desarrollo de plántula se analizaron mediante análisis de varianza (ANOVA) y comparación de medias con la prueba de Tukey $(p \leq 0.05)$. También se aplicó una prueba de Pearson $(p=0.10)$ para determinar la correlación entre variables morfológicas, germinación $y$ viabilidad. El paquete estadístico usado fue $R(R$ Core Team 2016).

\section{RESULTADOS}

\section{Variables morfométricas}

El análisis de varianza indicó diferencia significativa $(p<0.05)$ en el contenido de semillas por cápsula; las localidades Atetetla, Motuapa y los Amates presentaron los valores mayores $(175,210$ y 241 semillas, respectivamente; en tanto, Coacán (139) mostró la menor cantidad de semillas (Figura 1A y Figura 2). El porcentaje de semillas normales estuvo entre el 39.4 y $60.9 \%$ (Figura 1B), siendo inferior en Atetetla (34.9\%) y Coacán (46.7\%) y mayor en Motuapa $(60.9 \%)$; mientras que, la proporción de semillas estériles más baja la mostró Coacán (38\%), siendo las demás localidades, iguales entre sí (Figura 
A

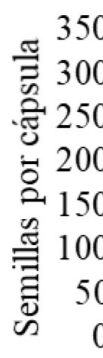

B

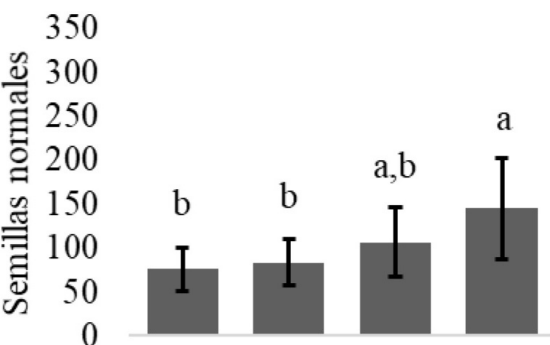

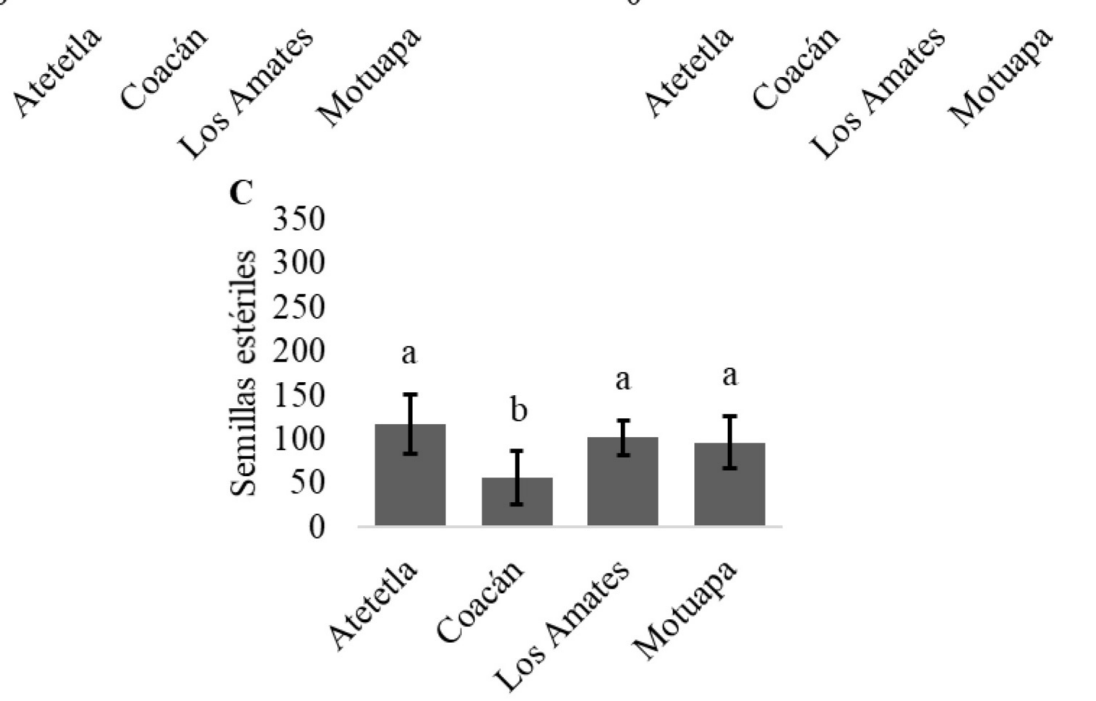

Figura 1. A) Contenido total de semillas por cápsula, B) semillas normales, C) semillas estériles de Agave angustifolia (Haw). Literales distintas son estadísticamente diferentes (Tukey, $\mathrm{p} \leq 0.05$ ). Las líneas indican la desviación estándar.

$1 C)$.

\section{Porcentaje de germinación y viabilidad}

Se observó que la germinación (Figura 3) fue mayor al $65 \%$, significativamente diferente $(p<0.05)$ en los sitios de colecta y Atetetla mostró el mayor valor (95\%). La prueba de viabilidad (Figura 4) con tetrazolio al $1 \%$ indicó que la proporción de semillas viables varió de acuerdo a los sitios de colecta, observando que Coacán presentó el menor porcentaje $(60.9 \%)$, estadísticamente diferente $(p<0.05)$ al de Atetetla (89.4\%).

\section{Desarrollo de plántula}

Las localidades mostraron un efecto significativa $(p<0.0001)$ sobre la altura de la planta y longitud de hoja, a los 6 meses de edad, la mayor altura de planta la mostró la semilla de Atetella $(3.7 \pm 0.2 \mathrm{~mm})$ y Motuapa $(3.2 \pm 0.2 \mathrm{~mm})$, mientras que la longitud de hoja mayor la mostró la semilla de Atetella $(10.8 \pm 0.2 \mathrm{~mm})$ (Tabla 2). En este mismo sentido, el tiempo de inmersión también mostró un efecto significativo sobre la altura de planta y longitud de hoja, observando que las plántulas que no se sometieron al tratamiento tuvieron mejor altura de planta $(3.6 \mathrm{~cm})$.

\section{Análisis de correlación}

Se encontró asociación positiva, entre la germinación y la viabilidad $(r=0.941)$, el largo de la hoja $(r=0.981)$ y ancho de la hoja $(r=0.907)$ a los seis meses después de la emergencia. La viabilidad, presentó alta correlación positiva con el desarrollo de la planta (altura de planta, largo y ancho de hoja) con coeficientes de correlación de 0.917, 0.918 y 0.996 , 


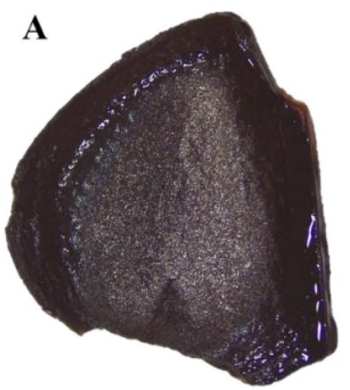

$\mathbf{C}$
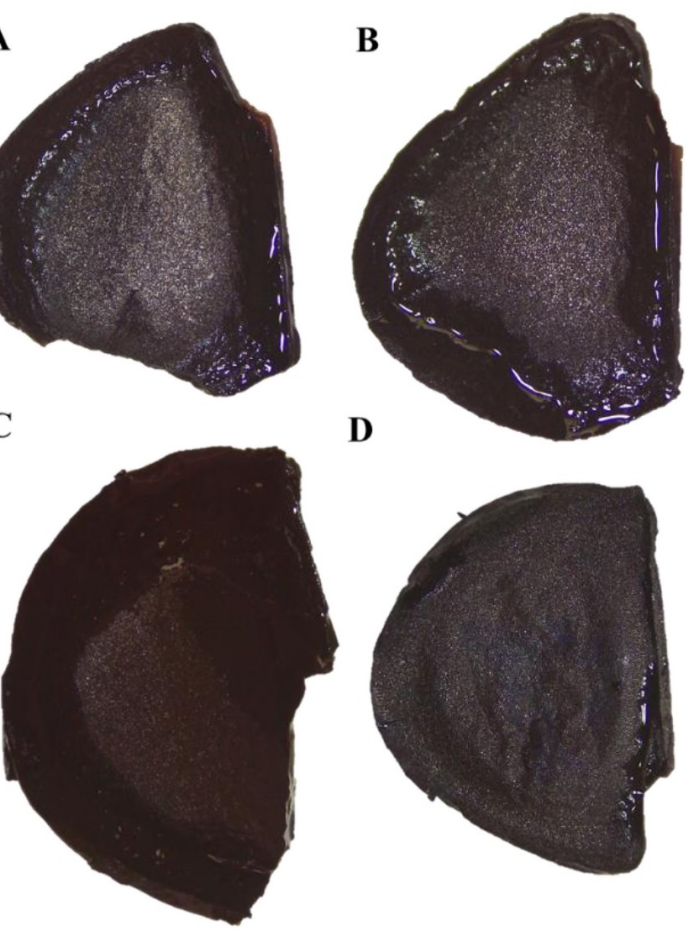

\section{$10 \mathrm{~mm}$}

Figura 2. Semillas de A. aungustifolia Haw. A) Atetetla; B) Coacán; C) Los Amates y D) Motuapa.

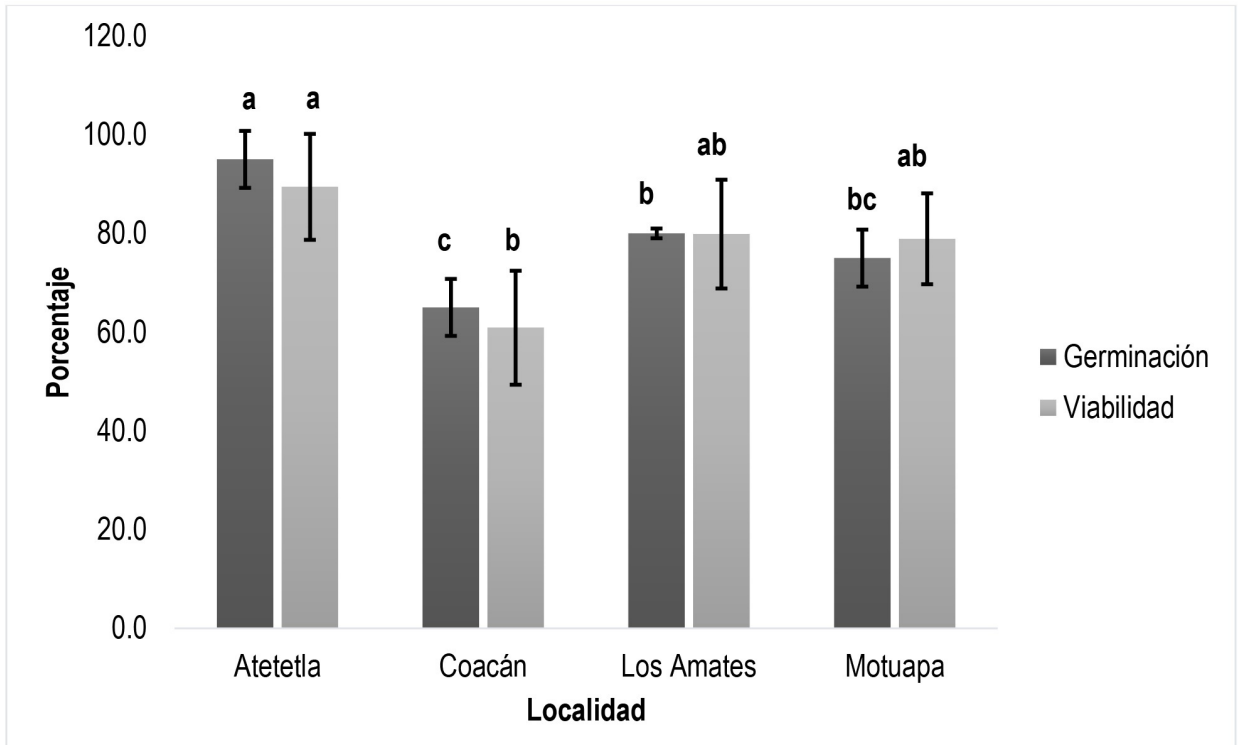

Figura 3. Porcentaje de germinación y viabilidad en semillas de $A$. angustifolia (Haw). Medias con literales distintas, por color de barra, son diferentes estadísticamente (Tukey, $p \leq 0.05$ ). Las líneas indican la desviación estándar. 
a)

a)

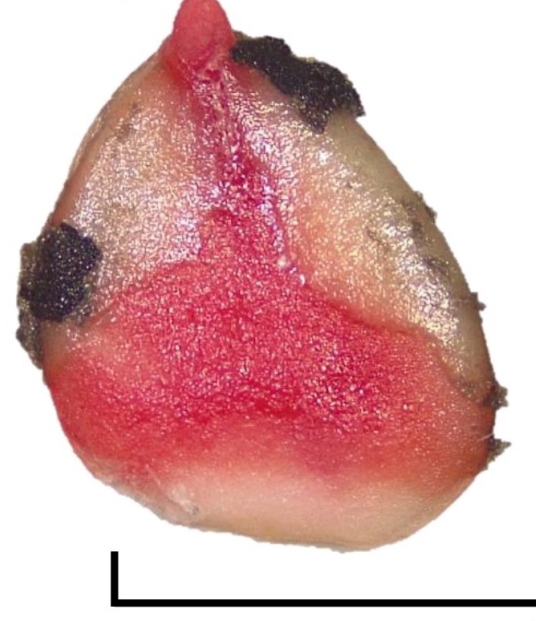

b)

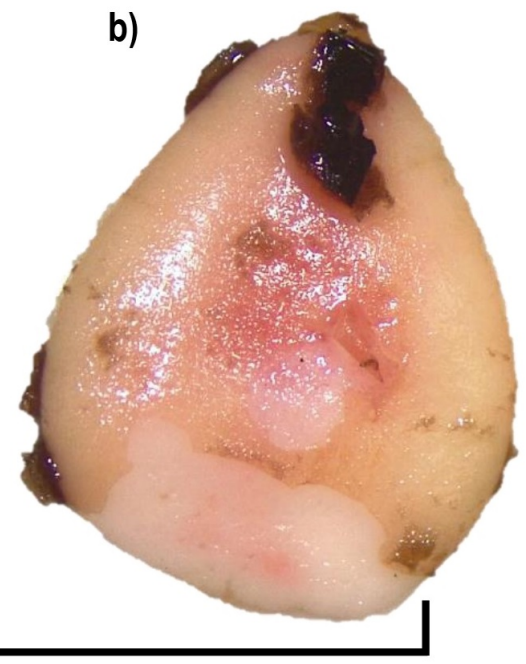

$10 \mathrm{~mm}$

Figura 4. Tinción de semillas de $A$. angustifolia (Haw) con Tetrazolio al 1\%. a) semilla viable; b) semilla no viable.

Tabla 2. Desarrollo de plántula de $A$. aungustifolia Haw. de semillas colectadas en cuatro localidades y diferentes tiempos de inmersión en agua.

\begin{tabular}{|c|c|c|c|c|c|c|c|c|c|}
\hline \multirow[b]{2}{*}{$\begin{array}{l}\text { Localidad / Tiempo después } \\
\text { de la siembra (meses) }\end{array}$} & \multicolumn{3}{|c|}{ Altura de planta $(\mathrm{cm})$} & \multicolumn{3}{|c|}{ Longitud de hoja (cm) } & \multicolumn{3}{|c|}{ Ancho de hoja $(\mathrm{cm})$} \\
\hline & 2 & 4 & 6 & 2 & 4 & 6 & 2 & 4 & 6 \\
\hline Atetetla & $1.7 \pm 0.2^{a}$ & $2.3 \pm 0.2^{a}$ & $3.7 \pm 0.2^{a b}$ & $4.3 \pm 1.0^{a}$ & $7.6 \pm 0.5^{a}$ & $10.8 \pm 0.2^{a}$ & $0.8 \pm 0.2^{a}$ & $1.0 \pm 0.2^{a}$ & $2.4 \pm 0.2^{a}$ \\
\hline Coacán & $1.2 \pm 0.0^{c}$ & $2.0 \pm 0.1^{b}$ & $3.0 \pm 0.0^{c}$ & $3.6 \pm 1.1^{a}$ & $6.5 \pm 0.3^{b}$ & $9.5 \pm 0.4^{c}$ & $0.5 \pm 0.1^{a}$ & $0.9 \pm 0.0^{a}$ & $2.0 \pm 0.4^{a}$ \\
\hline Los Amates & $1.3 \pm 0.3^{b}$ & $2.2 \pm 0.3^{a b}$ & $3.5 \pm 0.1^{b}$ & $4.1 \pm 1.3^{a}$ & $7.1 \pm 0.3^{a}$ & $10.0 \pm 0.3^{b}$ & $0.8 \pm 0.1^{a}$ & $1.1 \pm 0.1^{a}$ & $2.3 \pm 0.4^{a}$ \\
\hline $\begin{array}{l}\text { Motuapa } \\
\text { T Inmersión/ }\end{array}$ & $1.7 \pm 0.1^{a}$ & $2.2 \pm 0.2^{a b}$ & $3.8 \pm 0.2^{a}$ & $3.6 \pm 0.9^{a}$ & $7.3 \pm 0.3^{a}$ & $10.0 \pm 0.2^{b}$ & $1.0 \pm 0.7^{a}$ & $1.1 \pm 0.3^{a}$ & $2.3 \pm 0.3^{a}$ \\
\hline 0 & $1.5 \pm 0.2^{a}$ & $2.3 \pm 0.2^{a}$ & $3.6 \pm 0.4^{a}$ & $3.7 \pm 1.3^{a}$ & $7.1 \pm 0.5^{a}$ & $10.2 \pm 0.4^{a}$ & $0.6 \pm 0.1^{b}$ & $0.9 \pm 0.1^{a}$ & $2.2 \pm 0.3^{a}$ \\
\hline 24 & $1.5 \pm 0.3^{a b}$ & $2.1 \pm 0.2^{a}$ & $3.4 \pm 0.3^{b}$ & $3.9 \pm 1.0^{a}$ & $7.1 \pm 0.4^{a}$ & $9.9 \pm 0.7^{b}$ & $1.0 \pm 0.6^{a}$ & $1.1 \pm 0.2^{a}$ & $2.4 \pm 0.3^{a}$ \\
\hline $\begin{array}{l}48 \\
\text { Valor P }\end{array}$ & $1.4 \pm 0.4^{b}$ & $2.2 \pm 0.3^{a}$ & $3.5 \pm 0.3^{a b}$ & $4.0 \pm 1.0^{a}$ & $7.1 \pm 0.6^{a}$ & $10.1 \pm 0.4^{a b}$ & $0.8 \pm 0.1^{a b}$ & $1.1 \pm 0.2^{a}$ & $2.1 \pm 0.3^{a}$ \\
\hline Sitio & 0.0000 & 0.0424 & 0.0000 & 0.5356 & 0.0000 & 0.0000 & 0.1241 & 0.392 & 0.2141 \\
\hline Tratamiento & 0.0275 & 0.0587 & 0.0025 & 0.8664 & 0.9148 & 0.0431 & 0.0498 & 0.1711 & 0.2143 \\
\hline Interacción & 0.0000 & 0.0064 & 0.0003 & 0.5011 & 0.2071 & 0.0244 & 0.2814 & 0.5746 & 0.9344 \\
\hline CV & 4.26 & 8.26 & 3.1 & 19.55 & 5.25 & 2.57 & 24.25 & 21.38 & 16.89 \\
\hline
\end{tabular}

Media \pm desviación estándar de la media. Medias en sentido de las columnas que no comparten misma literal son diferentes estadísticamente (Tukey, $\mathrm{p} \leq 0.05)$. CV: Coeficiente de variación.

respectivamente $(p<0.10)$.

\section{DISCUSIÓN}

\section{Variables morfométricas}

En la Tabla 3 se muestra que el largo y ancho de cápsulas presentó diferencia estadística ( $p$ $<0.0001)$ en los diferentes sitios de colecta. Al respecto, Huerta-Lovera et al. (2018) señalan que el número de semillas por cápsula es independiente al sitio del crecimiento y periodo de maduración de la infrutescencia, observando valores de 0 a 294 semillas en $A$. salmiana, largos de entre 45.68 y $54.92 \mathrm{~mm}$, y ancho (diámetro) entre 20.15 y $22.5 \mathrm{~mm}$, valores similares a los encontrado en el presente estudio. Con respecto, al largo y ancho de semilla, las colectadas en Los Amates presentaron mayor longitud $\mathrm{y}$ anchura comparadas al resto de las colecciones (Figura 2), estadísticamente diferentes $(p<0.0001)$. Sobre lo mismo, Vázquez-Díaz et al. (2011) reportan en semilla de $A$. salmiana de San Luis Potosí, tamaños de semilla de 7.2 a $8.4 \mathrm{~mm}$ de longitud y 5.4 a $6.5 \mathrm{~mm}$ de ancho, valores que son menores a lo obtenido en el presente estudio. Lo cual puede deberse a la variedad y condiciones climáticas de la localidad donde se obtuvieron las colectas; como lo reportaron Fernández-Sosa et al. (2015) que en 
Tabla 3. Tamaño de semilla de A. aungustifolia Haw. colectadas en cuatro comunidades del estado de Guerrero.

\begin{tabular}{lcccc}
\hline \multirow{2}{*}{ Localidad } & LC & AC & LS & AS \\
\cline { 2 - 5 } & \multicolumn{4}{c}{ Cm } \\
\hline Atetetla & $5.37 \pm 0.50^{a}$ & $2.63 \pm 0.05^{a}$ & $0.82 \pm 0.07^{c}$ & $0.63 \pm 0.05^{c}$ \\
Coacán & $4.32 \pm 0.35^{b}$ & $2.40 \pm 0.26^{b}$ & $0.91 \pm 0.13^{b}$ & $0.70 \pm 0.09^{a b}$ \\
Los Amates & $5.10 \pm 0.40^{a}$ & $2.52 \pm 0.16^{a b}$ & $0.98 \pm 0.06^{a}$ & $0.73 \pm 0.05^{a}$ \\
Motuapa & $4.97 \pm 0.36^{a}$ & $2.35 \pm 0.07^{b}$ & $0.89 \pm 0.06^{b}$ & $0.66 \pm 0.06^{b c}$ \\
Valor F & 11.96 & 14.11 & 16.92 & 14.11 \\
Valor P & $<0.0001$ & $<0.0001$ & $<0.0001$ & $<0.0001$ \\
CV & 8.25 & 6.4 & 9.44 & 9.24 \\
\hline Media \pm desviación estándar. & Medias en sentido de las columnas, que no com- \\
parten misma literal son diferentes estadísticamente (Tukey, p $\leq$ 0.05). LC: largo \\
de cápsula; AC: ancho de cápsula; LS: largo de semilla; AS: ancho de semilla. CV: \\
coeficiente de variación.
\end{tabular}

semillas las localidades favorecieron la expresión del peso volumétrico. Mientras que, Garrido et al. (2005) mencionan que el tamaño de la semilla tiene una gran variación entre poblaciones, años y especialmente entre plantas.

En las variables de peso total y semillas normales, Los Amates, presentó los promedios más altos $(7.02$ y $2.24 \mathrm{~g}$, respectivamente); mientras que, Coacán fue la localidad donde se encontraron las cápsulas de menor peso y en Atetetla las de menor peso de semillas normales (Tabla 4). El tamaño de la semilla se relaciona directamente con la capacidad de reservar energía por parte de la semilla, por lo cual, puede ser un indicador de la calidad de la semilla, Respecto al peso de la cápsula y 1 000 semillas no se presentó diferencia estadística ( $p=0.073$ y $p=0.3789$, respectivamente) entre los sitios de colecta. En contraste, Vázquez-Díaz et al. (2011) reportaron en colectas de tres variantes de $A$. salmiana valores entre 10.8 y $14.5 \mathrm{mg}$ en peso de la semilla; mientras que, Hernández-Verdugo et al. (2010) mencionan que el peso de las semillas varía entre y dentro de las poblaciones.

\section{Porcentaje de germinación y viabilidad}

La capacidad germinativa en las semillas es afectada por factores intrínsecos como la especie y tamaño de la semillas y factores ambientales, como precipitación, temperatura, radiación solar y disponibilidad de agua, que afectan la planta madre y calidad de semillas en desarrollo (Ramírez-Tobías et al. 2012, Elizalde et al. 2017). Sobre esto, Ramírez-
Tobías et al. (2016) reportan en A. mapisaga $68 \pm$ $4 \%$ de semillas viables y $31 \pm 4 \%$ semillas inviables y en $A$. angustifolia subsp. tequiliana $53 \pm 6 \%$ y $19 \pm 3 \%$ de semillas viables e inviables, respectivamente. En A. potatorum Zucc., Langlé-Argüello et al. (2017) encontraron 85 y entre 66 - 77\% de viabilidad, en semillas almacenadas durante seis y 12 meses, respectivamente. Por lo que, la viabilidad es un indicador la actividad metabólica de los tejidos vivos e indirectamente es una estimación del poder germinativo (Copeland y McDonald, 2012).

\section{Desarrollo de plántula}

Los valores de altura de planta y longitud de hoja obtenidos difieren de los reportados por Bautista-Aparicio et al. (2017), quienes indican que a los seis meses las plántulas presentaron alturas entre 4.47 y $5.40 \mathrm{~cm}$ en $A$. potatorum Zucc. Lo que puede deberse a que el efecto de la fertilización que utilizaron influyó en el desarrollo de las plántulas, debido a que en el presente trabajo no se realizó fertilización. Con respecto a la longitud de hoja a los dos meses después de la emergencia, Atetetla presentó las hojas más largas $(4.3 \mathrm{~cm})$ y una longitud de hoja de $4.0 \mathrm{~cm}$ a un tiempo de inmersión de $48 \mathrm{~h}$. Valores similares a los reportados por Sánchez-Mendoza et al. (2017) que sometieron semillas de cuatro especies de Agave a tratamientos pregerminativos, con agua y reguladores de crecimiento, donde reportaron de 3.4 a $4.6 \mathrm{~cm}$. Al respecto, Peña-Valdivia et al. (2006) indican que el tamaño de semilla y la cantidad de agua absorbida influyen en la germinación de las 
Tabla 4. Peso de la cápsula y semillas de A. aungustifolia Haw. colectadas en el estado de Guerrero.

\begin{tabular}{lcccc}
\hline \multirow{2}{*}{ ocalidad } & PT & PC & PSN & P1000S \\
\cline { 2 - 5 } & \multicolumn{3}{c}{ G } \\
\hline Atetetla & $6.80 \pm 0.58^{a b}$ & $3.65 \pm 0.53^{a}$ & $1.67 \pm 0.20^{c}$ & $24.43 \pm 8.87^{a}$ \\
Coacán & $6.09 \pm 0.77^{b}$ & $3.01 \pm 0.25^{a}$ & $1.81 \pm 0.64^{b c}$ & $23.54 \pm 9.66^{a}$ \\
Los Amates & $7.02 \pm 0.94^{a}$ & $3.43 \pm 0.87^{a}$ & $2.24 \pm 0.30^{a b}$ & $24.01 \pm 10.97^{a}$ \\
Motuapa & $6.99 \pm 0.56^{a}$ & $3.24 \pm 0.26^{a}$ & $2.32 \pm 0.35^{a}$ & $18.10 \pm 6.41^{a}$ \\
Valor F & 3.52 & 2.52 & 6.16 & 1.06 \\
Valor P & 0.025 & 0.073 & 0.0018 & 0.3789 \\
CV & 10.87 & 16.25 & 10.02 & 15.53 \\
\hline
\end{tabular}

Media \pm desviación estándar. Medias en sentido de las columnas, que no comparten misma literal son diferentes estadísticamente (Tukey, $p \leq 0.05$ ). PT: peso total; PC: peso de cápsula; PSN: peso de semilla normales; P1000S: peso de mil semillas. CV: coeficiente de variación.

Tabla 5. Matriz de correlación entre las variables estudiadas de $A$. angustifolia Haw. colectadas en el norte de Guerrero.

\begin{tabular}{lccccccc}
\hline Variable & GE & V & LS & AS & AP & LH & AH \\
\hline V & $0.941^{*}$ & & & & & & \\
& $0.059^{\star \star}$ & & & & & & \\
AP & 0.731 & 0.917 & -0.287 & -0.49 & & & \\
& 0.269 & 0.083 & 0.713 & 0.51 & & & \\
LH & 0.981 & 0.918 & -0.687 & -0.783 & 0.725 & & \\
& 0.019 & 0.082 & 0.313 & 0.217 & 0.275 & & \\
AH & 0.907 & 0.996 & -0.374 & -0.552 & 0.947 & 0.883 & \\
& 0.093 & 0.004 & 0.626 & 0.448 & 0.053 & 0.117 & \\
\hline
\end{tabular}

${ }^{\star}$ Coeficiente de correlación de Pearson $(\mathrm{r}) .{ }^{*}$ Valores $\mathrm{P}(\leq 0.05) . \mathrm{GE}=$ germinación; $\mathrm{V}$ = viabilidad; $\mathrm{LS}$ = largo de semilla; $\mathrm{AS}$ = ancho de semilla; $\mathrm{AP}=$ altura de plántula; $\mathrm{LH}=$ longitud de hoja; $\mathrm{AH}=$ ancho de hoja.

semillas y emergencia de las plántulas.

\section{Análisis de correlación}

Se realizó una correlación (Pearson, $p=0.05$ ) y se encontró alta asociación positiva, entre la germinación y longitud a los seis meses después de la emergencia ( $r=0.981, p=0.019)$; así como, la viabilidad, con el ancho de hoja $(r=0.996, p=0.004)$ (Tabla 5). En A. potatorum Zacc., Langlé-Argüello et al. (2017) encontraron correlación positiva alta, entre germinación y viabilidad $(r=0.9027, p<0.05)$, y la longitud de planta con la germinación y viabilidad $(r=$ 0.8497 y $r=0.9208$, respectivamente) lo que sugiere que las semillas que presentan un mayor porcentaje de viabilidad y germinación desarrollan plántulas más vigorosas. Monroy-Vázquez et al. (2017) indican que la viabilidad y vigor son características indispensables en la germinación de las semillas y establecimiento de plántulas del género Opuntia.

\section{CONCLUSIONES}

Se presentó alta variabilidad en la morfología de las cápsulas y semillas, en la localidad de Atetetla se encontraron las cápsulas de mayor tamaño y peso. En los Amates se presentaron semillas de mayor tamaño y peso total. Los mayores pesos de semillas normales y 1000 semillas se encontraron en la localidad de Motuapa, la germinación y viabilidad en los sitios de colecta fue superior al $65 \mathrm{y}$ $89 \%$, respectivamente. La germinación y viabilidad de semilla presentaron correlación positiva con las variables de desarrollo de la plántula; por lo que, estas variables pueden ser un indicador de las características morfológicas de la planta y su producción mediante semilla es una alternativa viable. 


\section{LITERATURA CITADA}

Arzate-Fernández AM, Piña-Escutia JL, Norman-Mondragón TH, Reyes-Díaz JI, Guevara-Suárez KL, VázquezGarcía LM (2016) Regeneración de agave mezcalero (Agave angustifolia Haw.) a partir de embriones somáticos encapsulados. Revista Fitotecnia Mexicana 39: 359-366.

Barrientos-Rivera G, Esparza-lbarra EL, Segura-Pacheco HR, Talavera-Mendoza Ó, Sampedro-Rosas ML, Hernández-Castro E (2019) Caracterización morfológica de Agave angustifolia y su conservación en Guerrero, México. Revista Mexicana de Ciencias Agrícolas 10: 655-668.

Bautista Aparicio G, Sánchez Mendoza S, Bautista-Cruz MA (2017) Efecto de diferentes dosis de N y P en el crecimiento de Agave potatorum Zucc. Revista Mexicana de Agroecosistemas 4: 48-60.

Candia-Acosta JA, Díaz-Vásquez MA, Torres Herrera SI, Quiroz-Arratia JA, Dominguez-Calleros PA (2019) Germinación de semillas de Agave durangensis bajo diferentes coberturas en Durango, México. Revista Mexicana de Agroecosistemas 6: 18-27.

Copeland LO, McDonald MB (2012) Principles of seed science and technology. 4 ed. Ed. Springer Science \& Business Media. US. 467p.

Elizalde V, García JR, Peña-Valdivia CB, Ybarra MC, Leyva OR, Trejo C (2017) Viabilidad y germinación de semillas de Hechtia perotensis (Bromeliaceae). Revista de Biología Tropical 65: 153-165.

Esparza-Ibarra EL, Violante-González J, Monks S, Cadena-Iñiguez J, Araujo-Andrade C, Rossel-Kipping ED (2015) Los agaves mezcaleros del Altiplano Potosino y Zacatecano. En: Pulido-Flores G, Monks S, LópezHerrera M (ed) Estudios en biodiversidad. Vol I. University of Nebraska-Lincoln. EE.UU. pp: 227-245.

Fernández-Sosa R, Carballo-Carballo A, Villaseñor-Mir HE, Hernández-Livera A (2016) Calidad de la semilla de trigo de temporal en función del ambiente de producción. Revista Mexicana de Ciencias Agrícolas 6: 1239-1251.

Flores-Maya S, Vargas-Jurado MA, Suárez-Mota ME, Barrera-Escorcia H (2015) Karyotype analysis of Agave marmorata and Agave peacockii (Agavaceae) from alluvial terraces of the Zapotitlán river, Puebla, México. Polibotánica 40: 109-125.

García-Mendoza AJ (2007) Los agaves de México. Ciencias 87: 14-23.

Garrido JL, Herrera CM, Rey PJ (2005) Fuentes de variación en el tamaño de la semilla de la herbácea perenne Helleborus foetidus L. (Ranunculaceae). Anales del Jardín Botánico de Madrid 62: 115-125.

Hernández-Verdugo S, López-España RG, Porras F, Parra-Terraza S, Villarreal-Romero M, Osuna-Enciso T (2010) Variación en la germinación entre poblaciones y plantas de chile silvestre. Agrociencia 44: 667677.

Huerta-Lovera M, Peña-Valdivia CB, García-Esteva A, Kohashi-Shibata J, Campos-García H, Aguirre-Rivera JR (2018) Maguey (Agave salmiana) infructescence morphology and its relationship to yield components. Genetic Resources and Crop Evolution 65: 1649-1661.

Huerta-Zavala J, Sabino-López JE, Ochoa-Miranda R, Damián-Nava A, Segura-Pacheco HR, Hernández-Castro E (2019) Áreas potenciales para plantaciones de Agave angustifolia Haw en Guerrero, México. Agroproductividad 12: 3-9.

ISTA (2014) International rules for deed testing. The International Seed Testing Association (ISTA). Bassersdorf, $\mathrm{CH}$-Switzerland. pp: 15-70. 
Langlé-Argüello LA, Gutiérrez-Hernández GF, Martínez-Gutiérrez GA (2017) Efecto del tiempo de almacenamiento y procedencia de semillas de Agave potatorum Zucc. en la germinación y viabilidad. Revista Mexicana de Agroecosistemas 4: 1-7.

Maldonado-Peralta MA, De Los Santos-García G, García-Nava JR, Ramírez-Herrera C, Hernández-Livera A, Valdez-Carrazco JM, Corona-Torres T, Cetina-Alcalá VM (2016) Seed viability and vigour of two nanche species (Malpighia mexicana and Byrsonima crassifolia). Seed Science and Technology 44: 168-176.

Monroy-Vázquez ME, Peña-Valdivia CB, García-Nava JR, Solano-Camacho E, Campos H, García-Villanueva E (2017) Imbibición, viabilidad y vigor de semillas de cuatro especies de Opuntia con grado distinto de domesticación. Agrociencia 51: 27-42.

Ortiz-Hernández YD, Gutiérrez-Hernández GF, Corzo-Ríos LJ, García-Ramírez E, Martínez-Tomás SH (2018) Varietal and germinative characterization of Agave potatorum (Asparagaceae) seeds with different origins. Botanical Sciences 96: 628-639.

Peña-Valdivia CB, Sánchez-Urdaneta AB, Aguirre-Rivera JR, Trejo C, Cárdenas E, Villegas MA (2006) Temperature and mechanical scarification on seed germination of 'maguey' (Agave salmiana Otto ex Salm-Dyck). Seed Science \& Technology 34: 47-56.

R Core Team (2016) R: A language and environment for statistical computing. R Foundation for Statistical Computing, Vienna, Austria. https://www.R-project.org/. Fecha de consulta: 6 de agosto de 2020.

Ramírez-Tobías HM, Niño-Vázquez R, Aguirre-Rivera JR, Flores J, De Nova-Vázquez A, Jarquin-Gálvez R (2016) Seed viability and effect of temperature on germination of Agave angustifolia subsp. tequilana and $A$. mapisaga; two useful Agave species. Genetic Resources and Crop Evolution 63: 881-888.

Ramírez-Tobías HM, Peña-Valdivia CB, Aguirre-Rivera JR, Reyes-Agüero JA, Sánchez-Urdaneta AB, Valle GS (2012) Seed germination temperatures of eight Mexican Agave species with economic importance. Plant Species Biology 27: 124-137.

Ramírez-Tobías HM, Peña-Valdivia CB, Rogelio-Aguirre J (2014) Respuestas bioquímico-fisiológicas de especies de agave a la restricción de humedad. Botanical Sciences 92: 131-139.

Sánchez Mendoza S. Bautista-Cruz MA, Córdova Campos O, Crisanto-Martínez, M, Alavés-Jarquín F (2017) Tamaño de semilla y tratamientos pregerminativos en el crecimiento de agaves silvestres. Revista Mexicana de Agroecosistemas 4: 8-17.

Vázquez-Acosta A, Treviño-Carreón J, Estrada-Drouaillet B, Garcia-Alanis ML, Herrera Monsiváis MC, Tristán VV (2020) Morphological characterization of Agave tequilana Weber variety azul (Asparagaceae) under cultivation in the municipality of Llera, Tamaulipas, Mexico. Open Journal of Forestry 10: 264-275.

Vázquez-Díaz E, García-Nava JR, Peña-Valdivia CB, Ramírez-Tobías HM, Morales-Ramos V (2011) Tamaño de la semilla, emergencia y desarrollo de la plántula de maguey (Agave salmiana Otto ex Salm-Dyck). Revista Fitotecnia Mexicana 34: 167-173.

Villaseñor JL (2016) Checklist of the native vascular plants of México. Revista Mexicana de Biodiversidad 87: 559-902.

Zúñiga-Estrada L (2013) Nutrición de Agave tequilana y manejo de los fertilizantes en un sistema de producción intensiva (riego por goteo). Folleto Técnico. INIFAP-CIRNE. Villa Cuauhtémoc, Tamaulipas. 46p. 